\title{
Circulating Vascular Endothelial Growth Factor (VEGF) Levels in Advanced Stage Cancer Patients Compared to Normal Controls and Diabetes Mellitus Patients with Critical Ischemia
}

\author{
Yoka H. Kusumanto, Coby Meijer, Wendy Dam, Nanno H. Mulder and Geke A.P. \\ Hospers
}

Dept. of Medical Oncology, University of Groningen and University Medical Center Groningen, Groningen, The Netherlands.

\begin{abstract}
Anti-angiogenic therapy is emerging as a valuable tool in the treatment of patients with cancer. As VEGF is a central target in anti-angiogenic therapy, its levels in the circulation might be relevant in selecting tumor types or patients likely to respond to this treatment. Additional VEGF has been recognized as a key factor in the pathogenesis of diabetic retinopathy. Recently anti-angiogenic therapy has been advocated in this situation.

We measured VEGF levels in whole blood in 42 patients with high grade $(n=26)$ and low grade $(n=16)$ end stage cancer, and in 28 healthy controls and 37 patients with diabetes related vascular disease. Only 2/26 patients in the group of high grade cancer had significantly elevated VEGF levels, 1/16 in the low grade group and 1/28 in the healthy control group. In contrast, in 10/37 diabetic patients the mean VEGF levels were significantly elevated compared to the other groups. The mean level in these diabetic patients was significantly elevated compared to the other groups.

These data indicate the limitation of the use of circulating VEGF levels as a potential selection criterion for anti-angiogenic therapy in cancer patients and suggest further studies into its application in the management of diabetic complications.
\end{abstract}

Keywords: VEGF level, cancer, diabetes mellitus, Critical Limb Ischemia

\section{Introduction}

Anti-angiogenic therapy is emerging as an important strategy in the treatment of cancer (Ferrara, 2005). Following extensive in vitro and preclinical testing over many decades the therapeutic implications of tumor angiogenesis are finally having an impact in the clinic (Folkman, 1971). Up till now it is impossible to predict activity of anti-angiogenic therapy for particular tumor types or individual patients. However the favorable results of VEGF antibodies suggest that circulating levels of VEGF might give an indication of the potential of this treatment in tumors of different grades of malignancy or even in individual patients. A profile of elevated VEGF levels in the more aggressive cancers compared to slow growing tumors and in those tumor types known to respond to VEGF antibody therapy, would support further studies on VEGF levels as predictive markers.

Increased circulating VEGF levels have also been observed in patients with diabetes mellitus (Chiarelli et al. 2000; Valabhji et al. 2001). A variety of factors, implicated in the development of diabetic complications, have been shown to upregulate VEGF expression in vitro, including high glucose concentrations and advanced glycation end-products (Williams, 1997; Okamoto et al. 2002). Vascular proliferation is known to play a role in the development of diabetic retinopathy. It is therefore not surprising that developments in anti-angiogenic therapy in cancer have been closely followed in the field of ophthalmology. Preliminary evidence suggests that this treatment form either with bevacizumab or with its derivative ranibizumab is highly effective (Rosenfeld, 2005a; Rosenfeld, 2005b; Miller, 2005; Puliafito, 2005).

To further study the prevalence of elevated circulating VEGF levels in cancer patients and in diabetics we measured, in a population of patients who were referred with advanced stage cancer, the incidence of increased VEGF levels and compared them to values in the normal population and in a group of

Correspondence: G.A.P. Hospers, M.D., Ph.D., Dept. of Medical Oncology, University Medical Center Groningen, Hanzeplein 1, 9713 GZ Groningen, The Netherlands.

Tel: +31 50361 2821/1847; Fax: +31 50361 4862; Email: g.a.p.hospers@int.umcg.nl

Please note that this article may not be used for commercial purposes. For further information please refer to the copyright statement at http://www.la-press.com/copyright.htm 
non-cancer diabetic patients known to have severe ischemic vascular disease.

\section{Patients and Methods}

Forty-two patients who had incurable metastatic cancer and referred for palliative therapy were studied (median: 55 (range 19-75) years). These patients were separated in two groups, one with slow growing differentiated neuro-endocrine carcinoid tumors, comprising 16 patients, and a second group of aggressively progressive solid tumors of diverse origin, comprising 26 patients (Table 1). The 6 "others" in Table 1 are extragonadal germ cell tumor, metastatic insulinoom, myxoid solitary fibrous tumor, neuroendocrine pancreas tumor (no hormone producing), lung (non small cell) and testicular cancer.

Thirty seven diabetic patients (median: 71 (range 40-84) years of age, diabetic duration: median 16 years (range $0.5-55) \mathrm{HbA} 1 \mathrm{c}$ median: 7.6 (range 5.8-12.2)) with end stage vascular disease of the limbs were included. In the diabetic patient group the following investigations were performed: demographic characteristics such as age and diabetic duration and body mass index but also clinical assessment of edema were included. Concentrations of hemoglobin A1c (HbA1c), fasting glucose, cholesterol and triglycerides, C-reactive protein (CRP), creatinin, and albumin excretion ratio (two overnight urine collections) were measured. Standard laboratory assays were used. Fundus photographs of the retina were performed and graded as follows: no retinopathy, background retinopathy, pre-proliferative and

Table 1. Distribution of elevated VEGF levels.

\begin{tabular}{lcc}
\hline Subjects & n & $\begin{array}{l}\text { Elevated } \\
\text { VEGF level }\end{array}$ \\
\hline Controls & 28 & 1 \\
Carcinoid patients & 16 & 1 \\
Aggressive solid & 26 & 2 \\
tumor patients & & \\
- colon cancer & 8 & 0 \\
- breast cancer & 4 & 0 \\
- renal cancer & 3 & 0 \\
- melanoma & 5 & 0 \\
- others & 6 & 2 \\
Diabetes mellitus & 37 & $10^{*}$ \\
\hline
\end{tabular}

Number of patients with elevated VEGF levels (i.e. $>1200 \mathrm{pg} / \mathrm{ml}$; $95 \%$ confidence interval in healthy controls: $157.7-1200.0 \mathrm{pg} / \mathrm{ml}$ ).

*: $P=0.015$ by Mantel Haenzl chi square test. proliferative diabetic retinopathy. Ankle and toe pressures were measured according to conventional procedures using an $8 \mathrm{MHz}$ Parkes Doppler. Ankle to brachial index (ABI) and Toe to brachial index (TBI) were calculated as the quotient of absolute ankle and toe pressures to the simultaneously measured brachial pressure, respectively.

As a control population 28 healthy (median: 29 (18-54) years) volunteers were studied and randomly recruited from the medical and laboratory personal. Diabetic patients with evidence of systemic complications or systemic disease otherwise were excluded i.e. 1) proliferative eye disease, 2) history of malignancy or severe co-morbidity. The study was approved by the local Human Investigations Committee.

\section{VEGF measurements}

Venous blood was collected in sterile tubes containing CTAD (sodium citrate, theophylline, adenosine, dipyridamole, Becton Dickinson Vacutainer systems, France, Europe). Blood samples were diluted with two volumes of PBS (phosphate buffered saline) and subsequently lysed by freezing and thawing twice. Aliquots were stored at $-80^{\circ} \mathrm{C}$.

VEGF levels were determined in duplicate using the Quantikine human VEGF enzyme-linked immunosorbent assay (ELISA) (R \& D systems Inc. Minneapolis, MN). The minimum detection level was $9.0 \mathrm{pg} / \mathrm{ml}$ in whole blood as quoted by the manufacturer.

\section{Statistics}

The Kolmogorov-Smirnov test was used to confirm the assumption of normal distribution of VEGF samples in the healthy control group. The number of patients with elevated levels was compared between the groups using the mantel haenzl sqi square test. Mean VEGF levels were compared using the two sided student $\mathrm{T}$ test (unpaired). Statistical significance was set at $\mathrm{p}<0.05$.

\section{Results and Discussion}

Circulating VEGF mainly reflects VEGF derived from peripheral blood cells, including platelets and leucocytes. Therefore, we used whole blood for the measurement of VEGF, which contains all cell compartments, as was recommended previously (Salven et al. 1999). 
In the present study we found a $95 \%$ confidence interval of values in our control population $(n=28)$ between $157.71 \mathrm{pg} / \mathrm{ml}$ and $1200.03 \mathrm{pg} / \mathrm{ml}$. Individual VEGF levels above the upper limit of 1200 $\mathrm{pg} / \mathrm{ml}$ were considered to be elevated. Accordingly, in three out of 42 patients with advanced stage cancer VEGF levels were elevated, one patient with carcinoid cancer, one with an aggressive no hormone producing neuro-endocrine tumor and one with lung cancer, compared to one out of 28 cases in the control group and 10 out of 37 cases in the patients with diabetes $(p=0.015$, Table 1$)$.

In the cancer patients there was a tendency toward higher levels in patients with aggressive solid tumors compared to controls although this did not reach significance $(p=0.08$, Table 2$)$. There was no difference of occurrence of high levels of whole blood VEGF levels between aggressive and the more differentiated and slower growing carcinoid tumors. Also VEGF levels in the patients with colonic cancer, a tumor type that is accepted as an indication for angiogenic therapy did not exceed normal levels. As angiogenesis is necessary for tumor growth and the metastatic process, many attempts at quantifying this process have been made. Direct measurements include determination of micro vessel density in the tumor (Blann et al. 2002; Blann et al. 2001; Cascinu et al. 2000). However this method although effective in these studies in colorectal cancer requires tumor samples, limiting its applicability in the clinic as does immunohistochemistry on tumor cells or vessels. Indirect measurements include the various pro- or anti angiogenic factors in blood. Interestingly Tien et al. (Tien et al. 2006) found peripherically determined venous VEGF levels to be not inferior to levels downstream in the venous blood of gastro-intestinal tumors. Moreover VEGF levels correlated the most closely to patients clinico pathological characteristics.

A considerable number of studies have linked blood VEGF levels to tumor stage and prognosis in patients with cancer. Reports usually indicate that cancer patients tend to have higher levels of VEGF than controls, and that levels correlate with adverse prognostic factors. Subgroup analysis, for example comparing long-and short-term survivors, was suggestive for the existence of a relation of malignancy grade with VEGF levels. This effect was striking in an early study in lung cancer (Ohta et al. 1996). The same correlations were found in liver cancer, breast cancer and colon cancer (Torimura et al. 1998; Toi et al. 1995; Eppenberger et al. 1998; Gasparini et al. 1997; Takahashi et al. 1995; Cascinu et al. 2000; Ishigami et al. 1998). Yet, the question whether a relation can be found between VEGF levels and tumor stage has been answered equivocally in various studies. In renal cell cancer such a correlation was absent, but in planocellular esophagus cancer it was striking (Edgren et al. 2001; Wallner et al. 2001). The same was found in cervical cancer and differentiated thyroid cancer (Bachtiary et al.; Tuttle et al. 2002).

Usually it is assumed that tumor angiogenesis under the influence of elevated VEGF levels is the biological phenomenon involved. However the endpoint of that process, micro vessel density, was not always related to VEGF levels, even when these levels had been found to have predictive clinical relevance (Yudoh et al. 2001). In the latter study from Yudoh et al. in sarcoma patients, local relapse, metastatic progression and short survival were predicted by high tissue levels of VEGF but not with micro vessel density. In another study in bone sarcoma patients however, serum levels of VEGF were elevated in contrast to tissue levels in Ewing sarcoma (Holzer et al. 2001). On the other hand an apparent relation between tumor burden and circulating VEGF levels was shown by the rapid decrease of elevated levels after surgery for such different tumors as esophageal cancer and childhood Wilms tumor (McDonnell et al. 2001; Blann et al. 2001). Yet, in view of the rarity of

Table 2. VEGF whole blood levels.

\begin{tabular}{|c|c|c|c|c|c|}
\hline & Controls & All cancers & $\begin{array}{l}\text { Carcinoids } \\
\text { tumors }\end{array}$ & $\begin{array}{l}\text { Aggressive solid } \\
\text { mellitus }\end{array}$ & Diabetes \\
\hline $\begin{array}{l}\text { VEGF levels } \\
(\mathrm{pg} / \mathrm{ml}) \\
\text { P-value }\end{array}$ & $\begin{array}{l}491.7 \\
\pm 275.5\end{array}$ & $\begin{array}{l}592.6 \\
\pm 351.8 \\
\text { n.s. }\end{array}$ & $\begin{array}{l}525.6 \\
\pm 101.9 \text { n.s. } \\
\text { n.s. }\end{array}$ & $\begin{array}{l}634.1 \\
\pm 311.9 \\
\text { n.s.* }\end{array}$ & $\begin{array}{l}928.9 \\
\pm 443.2 \\
0.0001^{* *}\end{array}$ \\
\hline
\end{tabular}




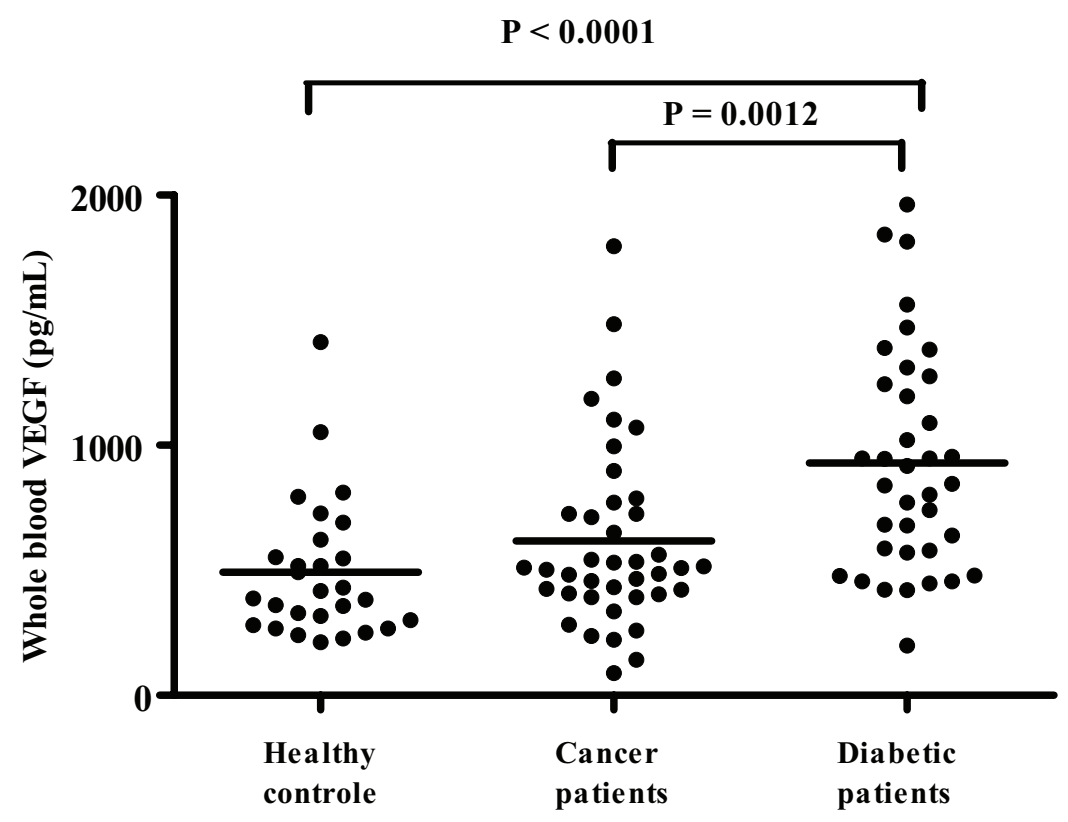

Figure 1. Mean VEGF levels. $P<0.05$ is considered significant (unpaired Student $T$ test).

increased VEGF levels found in our cancer patients it is doubtful that VEGF levels will become an important guideline in the treatment with antiangiogenic drugs on an individual or tumor type oriented basis.

As tissue anoxia is considered to be a major stimulus for VEGF production vascular insufficiency could also in non-malignant disease lead to increased VEGF levels (Maulik et al. 2000). For this purpose we studied a group of patients with end stage diabetic vascular insufficiency and found significantly elevated VEGF levels when mean VEGF levels ( $p=0.001$, Table 2 and Fig. 1$)$ as well as numbers of patients with elevated levels were compared. Although there was a trend for higher VEGF levels with duration of diabetes this did not reach significance $(\mathrm{p}=0.08$, data not shown). In addition we found no relation with other risk factors for diabetic complications such as, HbAlc, and albumin excretion ratio (AER), but also the degree of ankle edema, renal function, lipids, retinopathy and ABI/TBI were not related to circulating VEGF levels (data not shown). The role of VEGF in the development of diabetic vascular complications has become an increasingly intense studied subject in view of the rising prevalence of diabetes. Perhaps the strongest case for VEGF as a growth factor in diabetic vascular disease is proliferative diabetic retinopathy (Clermont et al. 1997; Malecaze et al. 1994). The results of studies on the relation with circulating VEGF levels were however not unequivocal with positive correlations found in proliferative retinopathy (Sydorova and Lee 2005; Chaturvedi, 2000). Our findings confirm that VEGF levels can be elevated in diabetes and that high levels are indeed common with end stage vascular disease as opposed to diabetics with healthy vessels (Chiarelli et al. 2000; Valabhji et al. 2001; Blann et al. 2002). The common occurrence of elevated VEGF levels in diabetic patients with vascular disease suggest that anti-angiogenic treatment directed at this growth factor is logical. This was already found to be successful in the treatment of diabetic retinopathy with ranibizumab, a derivative of bevacizumab. Interestingly, early clinical studies in the treatment of chronic ischemic limb disease with VEGF as a therapeutic agent, thus increasing endogenous VEGF levels, showed beneficial effects without causing severe adverse effects, even in diabetic patients (Baumgartner et al. 1998; Shyu et al. 2003).

In conclusion, our study indicates that the potential of the use of circulating VEGF levels as a selection criterion for anti-angiogenic therapy in cancer patients is limited. Significantly elevated VEGF levels in end stage solid tumor patients are rare $(2 / 26)$, such levels can be found also in healthy controls (1/28) and and most strikingly, in diabetic patients with ischemic vascular disease (10/37). Future studies should reveal the biologic relevance 
and hence the diagnostic and therapeutic implications for the treatment of vascular complications in diabetic patients.

\section{References}

Bachtiary, B., Selzer, E., Knocke, T.H. et al. 2002. Serum VEGF levels in patients undergoing primary radiotherapy for cervical cancer: impact on progression-free survival. Cancer Lett., 179:197-203.

Baumgartner, I., Pieczek, A., Manor, O. et al. 1998. Constitutive expression of phVEGF165 after intramuscular gene transfer promotes collateral vessel development in patients with critical limb ischemia. Circulation, 97:1114-23.

Blann, A.D., Belgore, F.M., McCollum, C.N. et al. 2002. Vascular endothelial growth factor and its receptor, Flt-1, in the plasma of patients with coronary or peripheral atherosclerosis, or Type II diabetes. Clin. Sci. (Lond)., 102:187-94.

Blann, A.D., Li, J.L., Li, C. et al. 2001. Increased serum VEGF in 13 children with Wilms' tumour falls after surgery but rising levels predict poor prognosis. Cancer Lett., 173:183-6.

Cascinu, S., Staccioli, M.P., Gasparini, G. et al. 2000. Expression of vascular endothelial growth factor can predict event-free survival in stage II colon cancer. Clin. Cancer Res., 6:2803-7.

Chaturvedi, N. 2000. Modulation of the renin-angiotensin system and retinopathy. Heart, 84 Suppl 1:i29-i31.

Chiarelli, F., Spagnoli, A., Basciani, F. et al. 2000. Vascular endothelial growth factor (VEGF) in children, adolescents and young adults with type 1 diabetes mellitus: relation to glycaemic control and microvascular complications. Diabet Med., 17:650-6.

Clermont, A.C., Aiello, L.P., Mori, F. et al. 1997. Vascular endothelial growth factor and severity of nonproliferative diabetic retinopathy mediate retinal hemodynamics in vivo: a potential role for vascular endothelial growth factor in the progression of nonproliferative diabetic retinopathy. Am. J. Ophthalmol., 124:433-46.

Edgren, M., Lennernas, B., Larsson, A. et al. 2001. Angiogenic factors: vascular endothelial growth factor (VEGF) and basic fibroblast growth factor (b-FGF) are not necessarily elevated in patients with advanced renal cell carcinoma. Anticancer Res., 21:1423-9.

Eppenberger, U., Kueng, W., Schlaeppi, J.M. et al. 1998. Markers of tumor angiogenesis and proteolysis independently define high- and low-risk subsets of node-negative breast cancer patients. J. Clin. Oncol., 16:3129-36.

Ferrara, N. 2005. VEGF as a therapeutic target in cancer. Oncology, 69 Suppl 3:11-6.

Folkman, J. 1971. Tumor angiogenesis: therapeutic implications. N. Engl. J. Med., 285:1182-6.

Gasparini, G., Toi, M., Gion, M. et al. 1997. Prognostic significance of vascular endothelial growth factor protein in node-negative breast carcinoma. J. Natl. Cancer Inst., 89:139-47.

Holzer, G., Obermair, A., Koschat, M. et al. 2001. Concentration of vascular endothelial growth factor (VEGF) in the serum of patients with malignant bone tumors. Med. Pediatr. Oncol., 36:601-4.

Ishigami, S.I., Arii, S., Furutani, M. et al. 1998. Predictive value of vascular endothelial growth factor (VEGF) in metastasis and prognosis of human colorectal cancer. Br. J. Cancer. 78:1379-84.

Malecaze, F., Clamens, S., Simorre-Pinatel, V. et al. 1994. Detection of vascular endothelial growth factor messenger RNA and vascular endothelial growth factor-like activity in proliferative diabetic retinopathy. Arch. Ophthalmol., 112:1476-82.

Maulik, N., Sasaki, H., Addya, S. et al. 2000. Regulation of cardiomyocyte apoptosis by redox-sensitive transcription factors. FEBS. Lett., 485:7-12.

McDonnell, C.O., Harmey, J.H., Bouchier-Hayes, D.J. et al. 2001. Effect of multimodality therapy on circulating vascular endothelial growth factor levels in patients with oesophageal cancer. Br. J. Surg., 88:1105-9.
Miller, J. 2005. MARINA Study Group. Randomized, controlled phase III study of ranibizumab (Lucentis) for minimally classic or occult neovascular age-related macular degeneration. Program and abstracts of the American Society of Retina Specialists 23rd Annual Meeting. July 16-20, Chicago, Illinois.

Ohta, Y., Endo, Y., Tanaka, M. et al. 1996. Significance of vascular endothelial growth factor messenger RNA expression in primary lung cancer. Clin. Cancer Res., 2:1411-6.

Okamoto, T., Tanaka, S., Stan, A.C. et al. 2002. Advanced glycation end products induce angiogenesis in vivo. Microvasc. Res., 63:186-95.

Puliafito, C.A. 2005. Lucentis for exudative AMD. Pharmacotherapy for macular diseases. Program and abstracts of the American Academy of Ophthalmology 109th Annual Meeting, October 15-18, Chicago, Illinois.

Rosenfeld, P.J. 2005a. Avastin for AMD. Retina 2005: Changing concepts and controversies. Program and abstracts of the American Academy of Ophthalmology 109th Annual Meeting. October 15-18, Chicago, Illinois.

Rosenfeld, P.J. 2005b. Systemic bevacizumab (Avastin) therapy for neovascular age-related macular degeneration (SANA) study: 12 week outcomes. Program and abstracts of the American Society of Retina Specialists 23rd Annual Meeting. July 16-20, Montreal, Canada.

Salven, P., Orpana, A., Joensuu, H. 1999. Leukocytes and platelets of patients with cancer contain high levels of vascular endothelial growth factor. Clin. Cancer Res., 5:487-91.

Shyu, K.G., Chang, H., Wang, B.W. et al. 2003. Intramuscular vascular endothelial growth factor gene therapy in patients with chronic critical leg ischemia. Am. J. Med., 114:85-92.

Sydorova, M., Lee, M.S. 2005. Vascular endothelial growth factor levels in vitreous and serum of patients with either proliferative diabetic retinopathy or proliferative vitreoretinopathy. Ophthalmic Res., 37:188-90.

Takahashi, Y., Kitadai, Y., Bucana, C.D. et al. 1995. Expression of vascular endothelial growth factor and its receptor, KDR, correlates with vascularity, metastasis, and proliferation of human colon cancer. Cancer Res., 55:3964-8.

Tien, Y.W., Chang, K.J., Chiu, Y.F., Huang, K.W., Lee, P.H. 2006. Comparison of angiogenic factor levels in tumor drainage and peripheral venous blood from colorectal cancer patients. Ann. Surg. Oncol., 13:1357-63.

Toi, M., Inada, K., Suzuki, H. et al. 1995. Tumor angiogenesis in breast cancer: its importance as a prognostic indicator and the association with vascular endothelial growth factor expression. Breast Cancer Res. Treat., 36:193-204.

Torimura, T., Sata, M., Ueno, T. et al. 1998. Increased expression of vascular endothelial growth factor is associated with tumor progression in hepatocellular carcinoma. Hum. Pathol., 29:986-91.

Tuttle, R.M., Fleisher, M., Francis, G.L. et al. 2002. Serum vascular endothelial growth factor levels are elevated in metastatic differentiated thyroid cancer but not increased by short-term TSH stimulation. $J$. Clin. Endocrinol Metab., 87:1737-42.

Valabhji, J., Dhanjil, S., Nicolaides, A.N. et al. 2001. Correlation between carotid artery distensibility and serum vascular endothelial growth factor concentrations in type 1 diabetic subjects and nondiabetic subjects. Metabolism., 50:825-9.

Wallner, G., Ciechanski, A., Dabrowski, A. et al. 2001. Vascular endothelial growth factor and basic fibroblast growth factor in patients with squamous cell oesophageal cancer. Folia Histochem Cytobiol, 39 Suppl 2:122-3.

Williams, B. 1997. Factors regulating the expression of vascular permeability/vascular endothelial growth factor by human vascular tissues. Diabetologia, 40 Suppl 2: S118-S120.

Yudoh, K., Kanamori, M., Ohmori, K. et al. 2001. Concentration of vascular endothelial growth factor in the tumour tissue as a prognostic factor of soft tissue sarcomas. Br. J. Cancer. 84:1610-5. 